\title{
Analytical Study of Reliable Multicast for Host Mobility in IP Networks ${ }^{1}$
}

\author{
Jiunn-Ru Lai and Wanjiun Liao \\ Department of Electrical Engineering \\ National Taiwan University \\ Taipei, Taiwan
}

\begin{abstract}
This paper studies analytically the performance of reliable multicast for host mobility in IP networks. In particular, we examine two schemes: global token rotating and local recovery with tunneling. Both schemes support reliable multicast for mobile hosts at the expense of extra buffer spaces in mobility agents. The token rotating mechanism uses a rotating token which reports global packet reception status for all the agents in the system to cope with host mobility. An agent cannot remove a packet out of the buffer unless all the mobile hosts in the global system have correctly received the packet. The local recovery with tunneling approach, on the other hand, relieves this global waiting constraint from each agent. It requires each mobile to be registered with one agent, but changeable to a different agent upon handoff. The packets not repairable by new agents are then tunneled from previous agents. Each agent cannot remove a packet unless all the registered hosts under its service have correctly received the packet. We model the

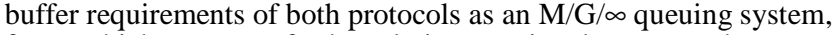
from which we can further derive service latency and system throughput. We have also conducted simulations to verify the derived analytical model. The results show that the local recovery with tunneling mechanism has much smaller buffer size, higher throughput, and lower service latency, as compared to the token rotating mechanism.
\end{abstract}

Keywords: Mobile reliable multicast, token rotating, RMMP

\section{INTRODUCTION}

IP multicasting is an efficient group communications mechanism, providing the same unreliable service as unicast IP transmission. Reliable multicast protocols [1][2][3][4][5] ensure end-to-end reliable reception of unreliable multicast datagrams for group members, assuming the existence of multicast delivery trees provided by underlying multicast routing protocols. Mobile reliable multicast adds the dimension of host mobility. With a mobile reliable multicast protocol, once a mobile has joined a multicast group, multicast datagrams will not be lost or duplicated due to roaming. Thus, the mobile is allowed to enjoy the service quality of reliable multicast as if it were a stationary host, and receives data streams continuously and reliably even while roaming.

This paper studies analytically the performance of reliable multicast for host mobility in IP networks. In particular, we examine two protocols: global token rotating [6] and local recovery with tunneling [7]. Both protocols support reliable multicast for mobile hosts at the expense of extra buffer spaces in mobility agents. The token rotating mechanism uses a rotating token which reports global packet reception status for all the agents in the system to cope with host mobility. An agent cannot remove a packet from the buffer unless all the mobile hosts in the global system have correctly received the packet. The local recovery with tunneling approach, on the other hand, relieves this global waiting constraint from each agent. It requires each mobile to be registered with one agent, but changeable to a different agent upon handoff. The packets not repairable by new agents are then tunneled from previous agents. Each agent cannot remove a packet unless all the registered hosts under its service have correctly received the packet. We model the buffer requirements of both protocols as an $\mathrm{M} / \mathrm{G} / \infty$ queuing system, from which we can further derive service latency and system throughput. We have also conducted simulations to verify the derived analytical model.

The rest of the paper is organized as follows. The analytical model is described in Section II. The performance results are shown in Section III, including the analytical and simulation results. Finally, the concluding remarks are included in Section IV.

\section{ANALYTICAL MODEL}

This section describes the analytical models of two protocols: the logical ring protocol [6] (denoted as Token Rotating (TR) protocol in the rest of the paper) and Reliable Mobile Multicast Protocol (RMMP) (i.e., local recovery with tunneling) [7]. It also examines the time until the last member in a group has successfully received a packet, and system throughput.

\section{A. Assumptions and Notations}

\footnotetext{
${ }^{1}$ This work is supported in part by the National Science Council, Taiwan, under Grant Number NSC 89-2219-E-002-004 and Grant Number NSC 89-E-FA06-2.
} 
The network topology we consider is a $D$-by- $D$ mesh with each cell (i.e., subnet) connected to four neighboring cells. Each cell has an agent associated with a multicast router. Suppose there is a single sender $\mathrm{X}$ multicasting packets to $\mathrm{N}$ identical mobile hosts distributed in the $D^{2}$ cells. Let $T_{\text {local }}$ be the average time to transmit a packet in one attempt in a cell, and $T_{\text {roam }}$, the average time a packet takes to travel one hop (i.e., from a cell to an adjacent cell).

We assume that the event that packets arrive in a cell (i.e., packet arrival to the buffer) is a Poisson distribution with a mean of $\lambda$. To focus on the impact of roaming on packet losses, which in turn affect buffer requirement, we assume no packet loss in the wired part of the network and all the losses are caused in the wireless part. Let the probability of a packet loss in the wireless channel be $p$. Selective repeat retransmission is assumed to recover lost packets in both protocols due to its well-known property of the highest throughput and the smallest buffer requirement. In addition, it is assumed enough buffers are available to hold all the packets in each agent. Thus, we can regard an infinite buffer in each agent. Depending on which protocol is used, a packet cannot be removed from an agent's buffer unless

(1) all the mobile hosts in the system have received it correctly (for TR).

(2) all the registered mobile hosts in the affiliated service area have received it correctly (for RMMP).

Thus, we can model the buffer of an agent as an $\mathrm{M} / \mathrm{G} / \infty$ queuing system. The packet arrival rate is $\lambda$; the packet service time has the same distribution as the time a packet is held in the agent's buffer. The buffer requirement $B$ of each protocol is then equal to the number of busy servers in the $\mathrm{M} / \mathrm{G} / \infty$ queuing system. $B=\lambda \times \bar{T}$, where $\bar{T}$ is the mean packet holding time in the buffer (i.e., the mean service time in the queuing system). Note that since TR does not allow mobile hosts to dynamically join or leave a group, for a fair comparison, we assume a closed network for both protocols. We make two additional assumptions: (1) all loss events in multicast datagrams at any host are mutually independent, and (2) no acknowledgements are lost.

\section{B Buffer Requirement}

Let $M_{r}$ be the number of transmission attempts for a packet to be received successfully by mobile host $r$. With a packet loss probability $p$ in the wireless channel, $M_{r}$ is geometrically distributed with a probability mass function as
$P_{M_{r}}(k)= \begin{cases}(1-p) p^{k-1}, & k=1,2,3, \ldots \\ 0 \quad, & \text { otherwise }\end{cases}$

Taking expectation, we obtain $\mathrm{E}\left[M_{r}\right]=1 / 1-p$.

Suppose that there are totally $R$ mobile receivers for each agent. Denote $M^{R}$ as the number of transmission attempts for a packet to be successfully received by all $R$ receivers. Thus, $M^{R}=\max _{r=1,2,3 \ldots R}\left\{M_{r}\right\}$.

Suppose the loss events among the $\mathrm{R}$ receivers are mutually independent. The probability cumulative function of $M^{R}$ is

$$
\begin{aligned}
& P\left(M^{R} \leq k\right)=P\left(\max _{r=1,2, \ldots, R}\left\{M_{r}\right\} \leq k\right) \\
& =\prod_{r=1}^{R} P\left(M_{r} \leq k\right)=\left(1-p^{k}\right)^{R} \quad, k=1,2,3, \ldots .
\end{aligned}
$$

Taking expectation of $M^{R}$, we have

$$
\begin{aligned}
E\left[M^{R}\right] & =\sum_{k=1}^{\infty} k P_{M^{R}}(k)=\sum_{k=1}^{\infty} P\left(M^{R} \geq k\right) \\
& =\sum_{k=1}^{\infty}\left[1-P\left(M^{R} \leq k-1\right)\right]=\sum_{k=1}^{\infty}\left[1-\left(1-p^{k-1}\right)^{R}\right]
\end{aligned}
$$

\section{1) Global Token Rotating Protocol}

The Token Rotating (TR) protocol [6] employs a global token rotating around all the cells in the $D$-by- $D$ mesh to cope with host mobility for reliable multicast in IP networks. In TR, a packet can be removed from the buffer only when all receivers in the global system have correctly received the packet. There are $\mathrm{N}$ mobiles in the system. In the steady state, it is assumed that the $\mathrm{N}$ mobiles are uniformly distributed in the $D^{2}$ cells. Thus, there are $N_{m}=N / D^{2}$ mobiles in each cell. Suppose that the source is located at cell $(m, n)$ in the $D$-by- $D$ mesh. A packet cannot be removed from the buffer of any agent unless the agent in the cell farthest (in hop count) from the source has successfully delivered the packet. Thus, the cell farthest from the source dominates the actual packet holding time in TR. In other words, the packet holding time in TR is roughly equal to the time between when a packet has been received by an agent in the cell farthest from the source and when all the mobiles under the service of the agent have correctly received the packet plus the token rotation overhead. 
Let $T_{\text {token }}$ denote the time a token rotates around all the $D^{2}$ cells. Thus,

$$
T_{\text {token }}=D^{2} \times T_{\text {roam }} \times \frac{\text { Token length }}{\text { Data packet length }}
$$

Let $T_{i}$ denote the mean packet holding time for the buffer in cell $i$. Averaging over $T_{i}, \forall i=1,2, \cdots D^{2}$, we obtain the average packet holding time in the buffer of each cell in TR as

$T_{T R}=E\left[M^{N_{m}}\right] \times T_{\text {local }}+Q_{(m, n)} \times T_{\text {roam }}+(0.5+0.5) T_{\text {token }}$

where $\quad Q_{(m, n)}=H_{(a, b)}-E\left[\sum_{\forall(x, y) \text { between }(m, n) \text { and }(a, b)} H_{(x, y)}\right]$,

$H_{(a, b)}$ is the distance between the source located at

point $(m, n)$ to the farthest cell located at point $(a, b)$ with $d[(m, n),(a, b)]=\max _{\substack{x=1,2,3 \ldots D \\ y=1,2,3 \ldots D}}\{|m-x|+|n-y|\}$.

$E\left[\sum_{\forall(x, y)} H_{(x, y)}\right]$ is the average distance to the source located at $(m, n)$ from any point located between the source at cell $(m, n)$ to the farthest cell at point $(a, b)$. Considering all possible points the source may be located, we have

$E\left[T_{T R}\right]=E\left[E\left[M^{N_{m}}\right] \times T_{\text {local }}+Q_{(m, n)} \times T_{\text {roam }}+T_{\text {token }}\right\rfloor$

$=E\left[M^{N_{m}}\right] \times T_{\text {local }}+E\left[Q_{(m, n)}\right] \times T_{\text {roam }}+T_{\text {token }}$

where (from Eq. 3)

$E\left[M^{N_{m}}\right]=\sum_{k=1}^{\infty}\left[1-\left(1-p^{k-1}\right)^{N_{m}}\right]$

According to [8], we obtain

$E\left[Q_{(m, n)}\right]= \begin{cases}\frac{1}{6 D}\left(5 D^{2}-6 D+4\right) & , \text { if } D \text { is even } \\ \frac{1}{6 D}\left(5 D^{2}-6 D+1\right) & , \text { if } D \text { is odd }\end{cases}$

Denote $B_{T R}$ as the mean buffer size of TR. Thus, $B_{T R}$ is

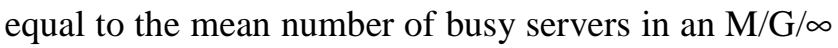
queuing system with mean service time $E\left[T_{T R}\right]$. $B_{T R}=\lambda \times E\left[T_{T R}\right]$.

\section{2) Local Recovery with Tunneling}

The Reliable Mobile Multicast Protocol (RMMP) [7] employs local recovery with tunneling to cope with host mobility. In RMMP, a mobile host is registered only with a single mobility agent, who serves as the reliability agent for the mobile as well. Each mobility agent in the affiliated cell maintains a buffer to provide reliable service for the registered mobiles under its service. A packet cannot be removed from the buffer unless all the registered receivers have acknowledged receptions. There are $\mathrm{N}$ mobiles in the system. Assume that in the steady state, the $\mathrm{N}$ mobiles are uniformly distributed in the $D^{2}$ cells. Thus, each agent provides service to $N_{m}=N / D^{2}$ mobiles. A packet can be removed from the buffer only when all the $N_{m}$ mobiles have correctly received the packet.

Let $T_{R M M P}$ denote the packet holding time in RMMP. Hence, $T_{R M M P}=M^{N_{m}} \times T$, where $M^{N_{m}}$ is the number of transmission attempts to correctly receive the packet for $N_{m}$ mobiles, and $T$ is the transmission time at each attempt. A mobile host may be staying in a cell, or moving from cell to cell during packet receptions. When a cascading handoff occurs during packet receptions, packets may be tunneled across multiple cells. Suppose that the probability a mobile moves out of range (i.e., roams to another cell) from a cell is $q$. Accounting for possible cascading handoffs, we have

$T=\sum_{k=0}^{\infty}(1-q) q^{k}\left(T_{\text {local }}+k \times T_{\text {roam }}\right)=T_{\text {local }}+\frac{q}{1-q} T_{\text {roam }}$

Taking expectation of $T_{R M M P}$, we obtain

$$
\begin{aligned}
& E\left[T_{R M M P}\right]=E\left[M^{N_{m}} \times T\right]=E\left[M^{N_{m}}\right] \times T \\
& =\sum_{k=1}^{\infty}\left[1-\left(1-p^{k-1}\right)^{N_{m}}\right] \times\left[T_{\text {local }}+\frac{q}{(1-q)} T_{\text {roam }}\right]
\end{aligned}
$$

Let $B_{R M M P}$ be the mean buffer size of RMMP. Thus, $B_{R M M P}$ is equal to the mean number of busy servers in an $\mathrm{M} / \mathrm{G} / \infty$ queuing system with mean service time $E\left[T_{R M M P}\right] . B_{R M M P}=\lambda \times E\left[T_{R M M P}\right]$

\section{Other Performance Metrics}

From Sec. 2.2, we can derive other performance metrics for both protocols, including the time until the last member in the system has successfully received a packet, and the system throughput. Due to space limitation, we skip the derivations and just present the results. Let $R_{(m, n)}$ be the distance (hop account) from a source located at the point $(m, n)$ to the farthest cell in the $D$-by- $D$ mesh network. For TR, the time until the 
last member to receive a packet is

$$
E\left[L R_{T R}\right]=E\left[M^{N_{m}}\right] \times T_{\text {local }}+E\left[R_{(m, n)}\right] \times T_{\text {roam }}
$$

where $E\left[R_{(m, n)}\right]= \begin{cases}\frac{1}{2}(3 D-2) & \text {,if } D \text { is even } \\ \frac{1}{2 D}(3 D+1)(D-1) & \text { if } D \text { is odd }\end{cases}$

And for RMMP, it is

$$
E\left[L R_{R M M P}\right]
$$

$=E\left[M^{N_{m}}\right] \times\left[T_{\text {local }}+\frac{q}{(1-q)} T_{\text {roam }}\right]+E\left[R_{(m, n)}\right] \times T_{\text {roam }}$ where $E\left[R_{(m, n)}\right]$ is the same as above.

\section{PERformanCE EVALUATION}

In this section, we examine the analytical results of both protocols. We have also conducted simulations to verify the derived analytical model. Note that the curves from simulation are mainly for reference, in an attempt to demonstrate the correctness of our analytical model. In the following, we will compare the performance of both protocols in terms of buffer size, service time for the last member, and throughput (scalability).

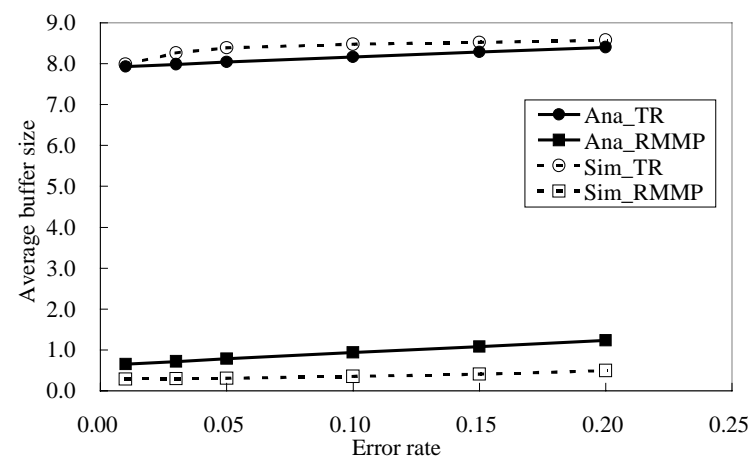

Figure 1. Error rate vs. buffer size

Figs. 1 to 4 show the mean buffer requirements of both protocols using both the derived analytical model and simulation. The solid lines are from the analytical model; the dash lines are from simulation. Fig. 1 shows the impact of the packet error rate in the wireless channel on mean buffer size. We fixed the following parameters for both the analytical model and the simulation: $\mathrm{N}=600, \mathrm{D}=100$, and $\mathrm{q}=0.1$. Both approaches have increasing mean buffer sizes as error rate increases. Compared to RMMP, TR requires much larger average buffer size due to its global token rotation mechanism.
Fig. 2 shows the impact of the mesh dimension on mean buffer sizes of both protocols. We fixed the following parameters: $\mathrm{N}=600, \mathrm{p}=0.1$, and $\mathrm{q}=0.1$. The average buffer size of TR increases linearly as mesh buffer size increases. This is because the cell farthest from the source determines the mean buffer size of the agents in TR. Thus, the packets in the cells nearest to the source cannot be removed unless the packets in the farthest cells can be removed. RMMP, on the other hand, relieves this global waiting constraint from the agents. Thus, it is invariant to mesh size and thus has a rather flat curve. In fact, the mean buffer size of RMMP decreases slightly as mesh size increases as shown in Fig. 2. This is because we have fixed mobiles for both approaches (due to assuming a closed network for the analysis. See Sec. 2.1.). According to [8],

$$
B_{R M M P} \cong O\left(\frac{p}{1-p} \log R\right)=O\left(\frac{p}{1-p} \log \frac{N}{D^{2}}\right)
$$

The larger the mesh size, the more cells in the system, and the fewer mobile hosts in each cell, resulting in smaller mean buffer requirement.

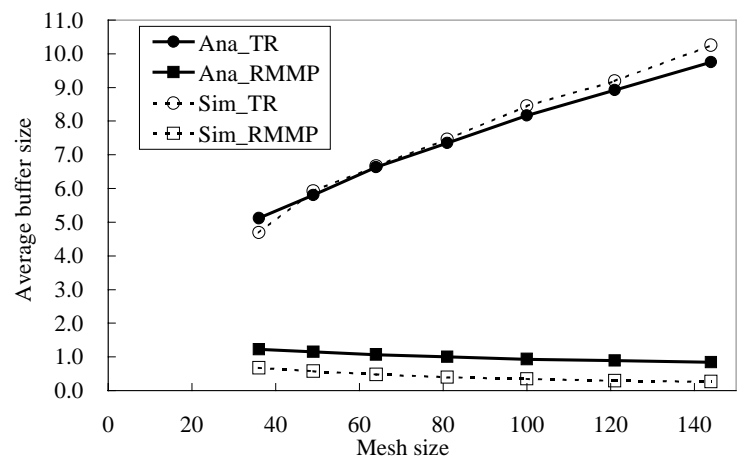

Figure 2. Mesh size vs. buffer size

Fig. 3 shows the impact of host mobility on the mean buffer sizes of both approaches. We fixed the following parameters: $\mathrm{N}=600, \mathrm{D}=100$, and $\mathrm{p}=0.1$. It can be observed that TR is invariant to host mobility because it uses the global token rotating mechanism to cope with packet losses due to host mobility. RMMP, on the other hand, makes use of local recovery and tunneling to deal with packet losses caused by roaming. Therefore, it needs a larger buffer as hosts move more frequently. Nevertheless, even under high mobility, the buffer requirement of RMMP is still much smaller than that of TR.

Fig. 4 shows the time until the last member in the system has successfully received a packet. It can be observed that the curves of the two protocols stay very close together. Note that Fig. 4 shows the finishing time 
of the last member in both protocols. Even if we consider the average service time of each packet in each protocol, according to Eqs. 4 and 5, RMMP still performs better.

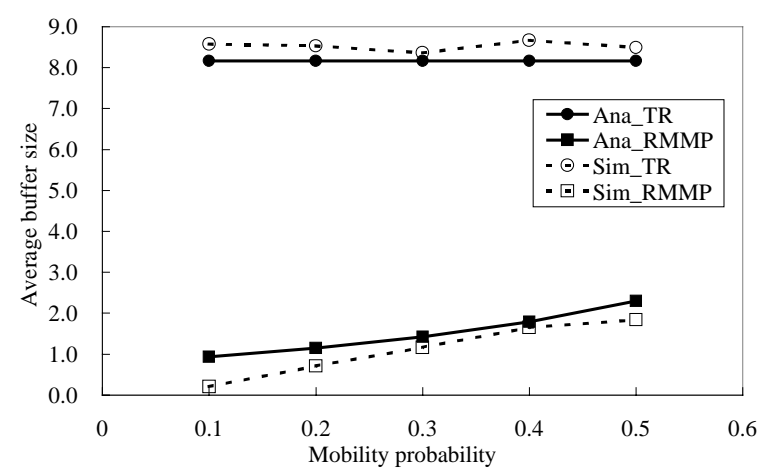

Figure 3. Mobility vs. buffer size

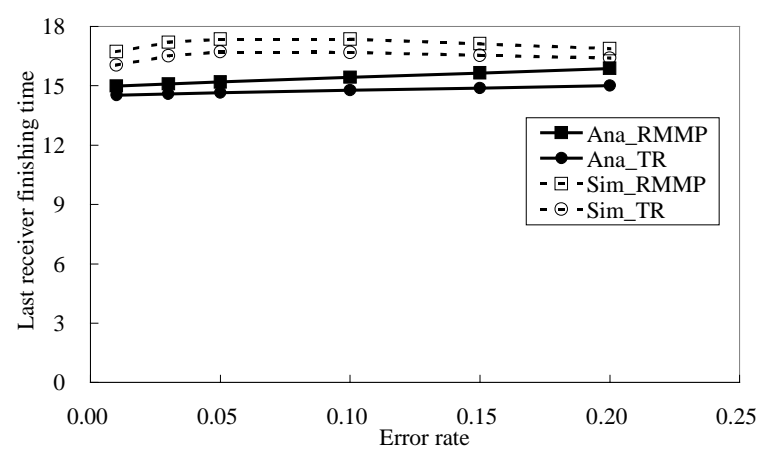

Figure 4. Error rate vs. last member finishing time

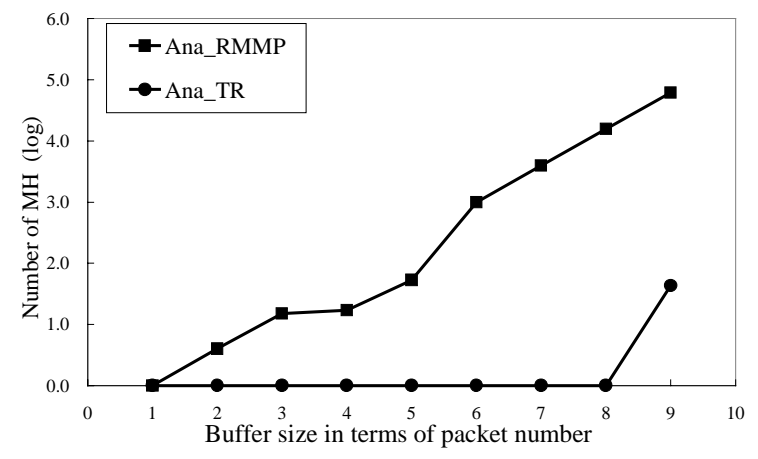

Figure 5. Scalability

Fig. 5 shows the scalability of both protocols in terms of the number of mobile hosts supported with any given buffer size. We varied the buffer size from 1 to 9 packets, but fixed $\mathrm{D}=100, \mathrm{~N}=600, \mathrm{p}=0.2$, and $\mathrm{q}=0.3$. It can be observed that RMMP scale better than TR even with very small buffer size. For example, with a buffer size less than 8 packets, say 5 packets, RMMP can support up to 100 mobile hosts in a cell, while TR is still unable to work with such small buffer size. The RMMP curve grows exponentially. With a buffer size of 8 , RMMP can support more than 10000 mobiles.

\section{CONCLUSION}

We have proposed an analytical model for reliable multicast protocols for host mobility in IP networks. We considered a global token rotating mechanism (i.e., TR) and a local recovery with tunneling (i.e., RMMP) mechanism, and modeled the buffer requirements of both protocols as an $\mathrm{M} / \mathrm{G} / \infty$ queue. We then compared both protocols using both the derived analytical model and simulation. The results have shown that RMMP has much better performance in terms of mean buffer requirements, mean packet holding time, and system throughput, as compared to the global token rotating mechanism.

\section{Reference}

[1] S. Floyd et al., "A Reliable Multicast Framework for Light-weight Sessions and Application Level Framing," IEEE/ACM Transaction on Networking, Dec. 1997, Vol.5, No. 6, pp. 784-803.

[2] Jo-Mei Chang and N. F. Maxemchuk, "Reliable Broadcast Protocols," ACM Transactions on Computer Systems, Vol. 1, No. 3, pp. 151-173, Aug. 1984.

[3] S. Paul et al, "Reliable Multicast Transport Protocol (RMTP)," IEEE Journal on Selected Areas in Communications, Vol. 15, No. 3, pp. 407-421, 1997.

[4] D. Li and D. R. Cheriton, "OTHER (On-Tree Efficient Recovery using Subcasting): A Reliable Multicast Protocol," in Proc. IEEE ICNP '99, pp. 237-245, Oct. 1998.

[5] C. Papadopoulos, G. Parulkar, and G. Varghese, "An Error Control Scheme for Large-Scale Multicast Applications," Proc. IEEE INFOCOM '98, pp. 1188-1196.

[6] Ioanis Kilolaidis and J. J. Harms, "A Logical Ring Reliable Multicast Protocol for Mobile Nodes," Proc. IEEE ICNP '99, Vancouver, Canada, pp. 106-113.

[7] Wanjiun Liao, Jen-an Ke, and Jiunn-Ru Lai, "Reliable Multicast with Host Mobility," in Proc. IEEE GLOBECOM 2000, San Francisco, CA, 2000.

[8] Jiunn-Ru Lai and Wanjiun Liao, "Performance Evaluation of Reliable Multicast Protocols for Host Mobility in IP Networks," Technical Report, Department of Electrical Engineering, National Taiwan University (TR-NTU-EE-S01-0323), 2001. 